\title{
Avaliação da disponibilidade de medicamentos genéricos em farmácias e drogarias de Maringá (PR) e comparação de seus preços com os de referência e similares
}

\author{
Wuelton Marcelo Monteiro', Gisely Cardoso de Melo' ${ }^{1}$, Gustavo Kiyoshi Massunari ${ }^{1}$, Dirce \\ Vendrametto Hübner², Raquel Soares Tasca ${ }^{2 *}$ \\ ${ }^{1}$ Faculdade de Farmácia da Universidade Estadual de Maringá, Paraná, ${ }^{2}$ Departamento de Farmácia e \\ Farmacologia, Universidade Estadual de Maringá, Paraná
}

${ }^{*}$ Correspondência:

R. S. Tasca

Departamento de Farmácia e

Farmacologia

Universidade Estadual de Maringá

Av. Colombo 5790-Jardim

Universitário

87020-900, Maringá - Paraná

E-mail: tasca@wnet.com.br
O debate sobre a implementação de genéricos vem sendo realizado no Brasil desde 1976. Entretanto, somente em 1991, foi elaborado um projeto, visando à implantação dos medicamentos genéricos. A lei $n^{\circ} 9787$ ("Lei dos genéricos") só foi publicada no Diário Oficial da União em 11 de fevereiro de 1999. Os objetivos deste trabalho foram verificar a disponibilidade de medicamentos genéricos em farmácias e drogarias, bem como comparar os preços destes medicamentos em relação aos similares e aos de referência. Verificou-se a disponibilidade dos medicamentos genéricos em 22 farmácias e drogarias do municipio de Maringá, estado do Paraná, de 30 de outubro a 22 de novembro de 2002. Dos 222 princípios ativos disponibilizados no Brasil como genéricos, foram encontrados 71 (32\%). Os medicamentos genéricos não sujeitos a controle especial são em média $42 \%$ mais baratos do que os de referência e $15 \%$ mais baratos que os similares. Os medicamentos genéricos de venda controlada com tarja vermelha são, em média, $36 \%$ mais baratos que os de referência e $12 \%$ mais baratos que os similares. Os medicamentos genéricos com tarja preta são, em média, 37\% mais baratos que os de referência e 14\% mais baratos que os similares.

\section{INTRODUÇÃO}

De acordo com a Resolução ${ }^{\circ} 10$ da Agência Nacional de Vigilância Sanitária (ANVISA), de 2 janeiro de 2001, medicamentos genéricos são aqueles que contêm o mesmo fármaco, na mesma dose, forma farmacêutica, indicação e via de administração de seu referente de marca, cujo prazo de proteção patentária expirou. A diferença entre os dois está na nomenclatura, pois os genéricos levam o nome do princípio ativo do medicamento, e o de marca adota o nome comercial (Mella, 2002). O medicamento genérico é designado conforme a DCB (Denominação Comum Brasileira) ou, na sua ausência, de acordo com a DCI (Denominação Comum Internacional), 
sendo identificado pela embalagem, que deve apresentar apenas o nome do princípio ativo e conter os dizeres: "Medicamento Genérico - Lei n ${ }^{\circ}$ 9787/99" - e uma tarja amarela, com um "G", em cor azul em destaque, conforme definição publicada na Resolução $n^{\circ} 47$, de 28 de março de 2001.

Além dos medicamentos genéricos, o mercado farmacêutico nacional conta com outras duas modalidades de medicamentos: os medicamentos de referência e os similares. Conforme a Lei 9787/99, medicamento de referência é definido como "produto inovador registrado no órgão federal responsável pela vigilância sanitária e comercializado no País, cuja eficácia, segurança e qualidade foram comprovados cientificamente junto ao órgão federal competente, por ocasião do registro". A mesma lei conceitua medicamento similar como "aquele que contém o mesmo ou os mesmos princípios ativos, apresenta a mesma concentração, forma farmacêutica, via de administração, posologia e indicação do medicamento de referência registrado no órgão federal responsável pela vigilância sanitária, podendo diferir somente em características relativas ao tamanho e forma do produto, prazo de validade, embalagem, rotulagem, excipientes e veículos, devendo sempre ser identificado por nome comercial ou marca" (Brasil, 1999).

Os medicamentos genéricos deverão passar por testes de bioequivalência e biodisponibilidade, tendo, assim, a garantia de que possuem mesmo valor terapêutico de um medicamento de marca. A Resolução ${ }^{\circ} 41$ da ANVISA, de 28 de abril de 2000, é que estabelece os critérios mínimos para a aceitação de unidades que realizam ensaios de equivalência farmacêutica, biodisponibilidade e bioequivalência. Conforme a resolução anterior, os testes envolvem a etapa de equivalência farmacêutica e as etapas clínica, analítica e estatística, devendo ser realizados em laboratórios credenciados. No Brasil, existem mais de vinte laboratórios preparados para realizar os testes, sendo na maioria laboratórios de universidades (Santos, 2000). Destes, 18 centros de biodisponibilidade e bioequivalência estão certificados pela ANVISA, sendo que 13 estão localizados no estado de São Paulo, dois em Goiás e um em cada um dos estados do Ceará, Pernambuco e Rio de Janeiro. Existem outros laboratórios em fase de certificação nos estados de Minas Gerais, Rio de Janeiro, Rio Grande do Sul e São Paulo (ANVISA, 2005).

O medicamento similar é uma opção de comercialização definida estrategicamente pela empresa produtora e não pode ser utilizada para gerar confusão nos profissionais de saúde e nos pacientes, devendo ser apresentado com nome de fantasia (Jatene et al., 2002). A Resolução no ${ }^{\circ} 134$, de 29 de maio de 2003, foi elaborada devido à necessidade de comprovação da eficácia e da segurança dos produtos similares. Segundo a nova legislação, a garantia da qualidade de um medicamento similar deve compreender estudos controlados, envolvendo ensaios de equivalência farmacêutica e biodisponibilidade relativa.

A consolidação do mercado de genéricos, no Brasil, representa importante estratégia governamental, uma vez que significa maior acesso da população aos medicamentos (Valente, Storpirtis, 2001). Segundo Barros (1997), apenas $23 \%$ da população brasileira tem acesso aos medicamentos e esta pequena parcela está sujeita a muitos problemas. O surgimento dos medicamentos genéricos se deu devido a esta dificuldade, trazendo esperanças ao quadro desanimador da saúde brasileira (Santos, 2000).

O difícil acesso aos medicamentos não é apenas privilégio de outros países em desenvolvimento (Chirac, 1993), pois o que acontece no panorama da saúde brasileira não é diferente (Lyra Junior et al., 1999). A camada economicamente privilegiada possui poder para comprar, e até abusar destes insumos, enquanto que a maioria da população ainda sofre com a falta de acesso, dependendo exclusivamente dos programas governamentais para o fornecimento dos medicamentos (Barros, 1995). Os medicamentos genéricos se inserem, portanto, como ferramenta fundamental na racionalização do uso de medicamentos.

O debate sobre a implementação de genéricos vem sendo realizado, nos países desenvolvidos, pela Organização Mundial da Saúde (OMS), desde 1958. Em conseqüência, nos Estados Unidos, aproximadamente 9 mil medicamentos genéricos já estão no mercado e muitos outros esperam aprovação da Food and Drug Administration (FDA). Naquele país, a substituição por genéricos vem aumentando, a cada ano, com mais de um bilhão de prescrições, ou seja, cerca de metade das prescrições dispensadas (Sax, 2000). No Brasil, o assunto vem sendo discutido desde 1976. Entretanto, somente em 1991 foi elaborado um projeto, tendo como meta a implantação dos medicamentos genéricos. A Lei n ${ }^{\circ}$ 9787, mais conhecida como "Lei dos Genéricos" só veio a ser publicada no Diário Oficial da União (DOU) em 11 de fevereiro de 1999 (Brasil, 1999).

A política de medicamentos genéricos, ao ser definitivamente implementada, com seriedade e respeitando um rigoroso controle de qualidade, gera muitas vantagens, entre outras: medicamentos de melhor qualidade, mais seguros e eficazes, comprovados pela realização de testes de equivalência farmacêutica e bioequivalência (Barros, 1995) e de menor preço, pois os fabricantes ao produzí-los não fazem investimentos em pesquisa e nem em propaganda. Para adoção desta política, envolvendo a produção, a garantia de qualidade, prescrição e dispensação, é de fundamental importância a participação ativa e consciente dos profissionais responsáveis pela prescrição e dispensação destes medicamentos. Neste contexto, o farmacêutico 
deveria ser o principal conhecedor no que tange os medicamentos e deve estar muito bem atualizado e instruído para proceder à intercambialidade ou substituição dos medicamentos de referência por genéricos, com eficiência e credibilidade (Santana et al., 2003).

Segundo Santos (2000), a sociedade também é exigida com a política dos genéricos, no sentido de aprender para se defender e cobrar os seus direitos. Os benefícios que os genéricos trazem devem ser divulgados, pois aumentam as chances de a população realizar o tratamento farmacológico completo. Na opinião de Serra (2000a), a dificuldade é que muitos médicos ainda não receitam os genéricos e os pacientes não sabem da possibilidade de utilizá-los. Para que esta política seja bem sucedida, tornase imprescindível a participação destes profissionais, pois além da prescrição eles podem disseminar informações sobre os medicamentos genéricos à população.

O uso do nome genérico oferece dois benefícios fundamentais: possibilita a todos os envolvidos, tanto profissionais da saúde como pacientes, a informação isenta sobre o medicamento, não se limitando a material de propaganda, que na realidade tem sido responsável pela educação continuada dos profissionais. Além disso, possibilita ao consumidor identificar os produtos, fomentando a concorrência e resultando em queda dos preços (Zanini, 1999).

Os laboratórios não são obrigados a produzir os genéricos, mas de acordo com Serra (2000b), as farmácias e drogarias são obrigadas a oferecê-los e poderão fechar se não os tiverem. Para ele existe má vontade por parte dos laboratórios e grandes redes de farmácia em produzir e comercializar genéricos. Suspeita-se de um boicote por parte dos estabelecimentos, devido à pequena quantidade destes produtos nas prateleiras de farmácias e drogarias. No entanto, parece haver laboratórios e indústrias interessadas em produzir genéricos. Em Goiás, 13 indústrias já estão instaladas e em fase de ampliação (Brandão, 2000).

A combinação entre eficiência na fiscalização por parte do governo, engajamento dos médicos, interesse da indústria nacional ou de outro país que produza genérico, abastecimento adequado das farmácias e aceitação da população fará com que cada vez mais o medicamento genérico se torne a melhor opção de mercado (Jatene et al., 2002).

Dessa forma, faz-se necessário verificar o preço dos medicamentos genéricos e a sua disponibilidade em farmácias e drogarias, considerando que a implantação de medicamentos genéricos no País ocorreu com o intuito de que a população tivesse acesso aos medicamentos, com menor custo (Marques, 2001).

Os objetivos deste trabalho foram verificar a disponibilidade de medicamentos genéricos não sujeitos a controle especial, de venda controlada com tarja vermelha e com tarja preta nas farmácias e drogarias, bem como comparar os preços dos medicamentos genéricos em relação aos medicamentos similares e aos de referência.

\section{MATERIAIS E MÉTODOS}

Para desenvolvimento desta pesquisa os dados foram coletados nas farmácias e drogarias do município de Maringá, estado do Paraná, no período de 30 de outubro a 22 de novembro de 2002. Foram sorteadas aleatoriamente $30 \%$ das farmácias e drogarias, sendo que 22 estabelecimentos farmacêuticos fizeram parte da pesquisa, dos quais 10 estavam localizados na região central (representando $30 \%$ dos estabelecimentos dessa região), enquanto 12 estavam localizados na periferia (representando 30\% dos estabelecimentos dessa região). Essa seleção atendeu a um critério que buscou representar uniformemente a distribuição de farmácias que atendem à população de Maringá.

Os medicamentos foram divididos em três grupos: medicamentos não sujeitos a controle especial, de venda controlada com tarja vermelha e de venda controlada com tarja preta. A seleção destes medicamentos foi realizada por meio da Lista de Genéricos elaborada pela ANVISA e publicada no Diário Oficial da União (DOU) de 27 de setembro de 2002, cuja seleção incluiu todos os medicamentos genéricos desta lista.

\section{Disponibilidade dos medicamentos}

Para certificar a existência dos medicamentos genéricos nos estabelecimentos comerciais farmacêuticos, técnicos da Proteção e Defesa do Consumidor (PROCON) e estagiários da disciplina de Estágio Supervisionado para Farmacêutico do curso de Farmácia da Universidade Estadual de Maringá (UEM - PR) verificaram nas farmácias e drogarias a disponibilidade do medicamento genérico. Foi considerado ausente o medicamento genérico cujo princípio ativo não era disponibilizado em nenhuma de suas apresentações nos 22 estabelecimentos pesquisados.

\section{Comparação dos preços}

O preço do medicamento genérico foi registrado para posterior comparação frente aos dos medicamentos de referência e similares, que também foram anotados. A informação sobre os preços destes medicamentos foi obtida pelo valor definido pelo estabelecimento farmacêutico para determinada especialidade farmacêutica.

A definição dos preços dos medicamentos para o consumidor é realizada considerando a Lei n ${ }^{\circ}$ 10147, de 21 
de dezembro de 2001, que dispõe sobre a forma de definição do preço do fabricante e do preço máximo ao consumidor dos medicamentos determinando, assim, as margens de comercialização desses produtos (Indifarma, 2003). Existem publicações que divulgam os preços máximos ao consumidor e servem como sugestão de venda. Uma dessas publicações, o Índice de Preços Farmacêuticos (Inditec), foi o único encontrado nos estabelecimentos farmacêuticos que fizeram parte desta pesquisa. $\mathrm{O}$ Inditec é uma publicação quinzenal, cuja editoração é feita por INDITEC Índices Técnicos e Processamento Ltda (Maringá, Paraná) e apresenta sugestões de vendas calculadas a partir de listagens fornecidas pelos laboratórios fabricantes (Indifarma, 2003). Com base no Inditec, as farmácias e drogarias elaboram listas próprias dos preços dos seus produtos, os quais são repassados ao consumidor. Estes preços foram os fornecidos pelos estabelecimentos farmacêuticos e anotados para a presente pesquisa.

A comparação foi feita por meio da diferença percentual de preços entre os medicamentos genéricos, similares e de referência. Essa diferença foi calculada após a obtenção dos preços nas farmácias e drogarias ao final da pesquisa, sendo que os valores apresentados no presente trabalho correspondem às médias das diferenças obtidas nos diferentes estabelecimentos.

A apresentação (forma farmacêutica e posologia) escolhida como padrão para as comparações foi aquela cujo princípio ativo disponibilizado como medicamento genérico era única ou a mais freqüente no momento da análise dos dados. Assim, quando um estabelecimento possuía medicamentos genéricos de diferentes fabricantes e apresentações, eram anotados os preços de todas as especialidades farmacêuticas e, posteriormente, na análise dos dados, era escolhida a mais freqüente para a comparação dos preços. Os medicamentos similares e de referência escolhidos para a comparação de preços foram aqueles com a mesma apresentação dos medicamentos genéricos. No caso da existência de medicamentos similares de diferentes marcas no mesmo estabelecimento, com a mesma apresentação do genérico correspondente, coletava-se o preço de todos e obtinha-se a média. Foram admitidos, dessa forma, similares de diferentes marcas para o cálculo das diferenças percentuais entre as categorias de medicamentos.

\section{RESULTADOS E DISCUSSÃO}

\section{Quanto à disponibilidade}

Dos 222 princípios ativos disponibilizados no Brasil como especialidades farmacêuticas genéricas, foram en- contrados 71 princípios ativos (32\%), segundo Lista de Genéricos divulgada pela ANVISA/2002. Os medicamentos não sujeitos a controle especial encontrados representaram $22 \%$ do total de medicamentos. Os medicamentos de venda controlada, com tarja vermelha e preta, representaram $70 \%$ dos medicamentos genéricos destes grupos liberados no Brasil.

Conforme a Figura 1 verifica-se que 36\% dos estabelecimentos dispõem de até 20 medicamentos genéricos não sujeitos a controle especial em suas prateleiras. Os outros 64\% das farmácias e drogarias não dispõem de medicamento genérico não sujeito a controle especial constante na lista de genéricos divulgada pela ANVISA.

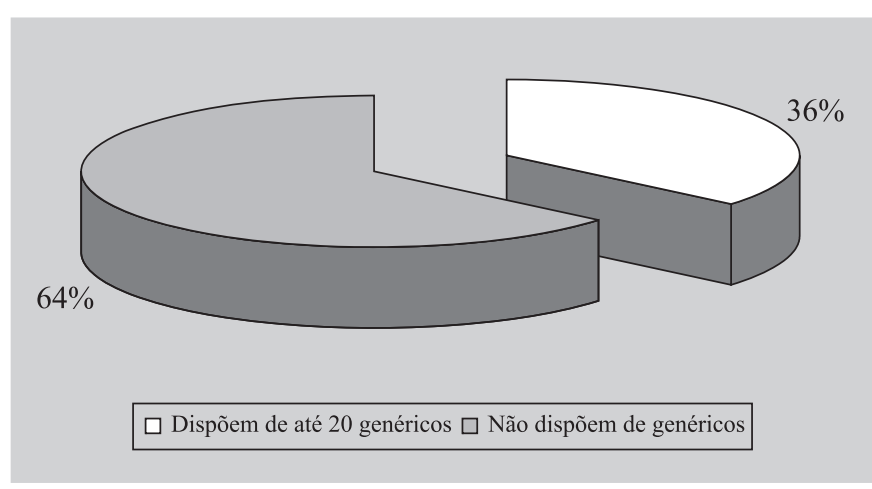

FIGURA 1 - Disponibilidade de medicamentos genéricos não sujeitos a controle especial em farmácias e drogarias no município de Maringá-PR, no período de 30/10 a 22/11 de 2002.

A respeito dos medicamentos genéricos de venda controlada com tarja vermelha, $14 \%$ dos estabelecimentos possuem de 9 a 14 medicamentos genéricos de um total de 14 medicamentos constantes da lista, de acordo com a Figura 2. Observa-se, ainda, que dos outros $86 \%$ dos estabelecimentos, $36 \%$ dispõem de até 7 medicamentos. O restante das farmácias e drogarias, isto é, $50 \%$ não possuem qualquer dos medicamentos genéricos controlados de tarja vermelha pesquisados.

Observa-se, na Figura 3, que $27 \%$ dos estabelecimentos possuem de 3 a 7 medicamentos controlados com tarja preta de um total de 7 medicamentos da lista. Os outros $73 \%$ dos estabelecimentos farmacêuticos dispõem de 1 ou 2 medicamentos de um total de 7 . Todos os princípios ativos disponibilizados como genéricos com tarja preta, constantes na lista divulgada pela ANVISA, foram encontrados no presente trabalho.

\section{Quanto ao preço}

Neste trabalho, verificou-se que os medicamentos genéricos não sujeitos a controle especial são, em média 


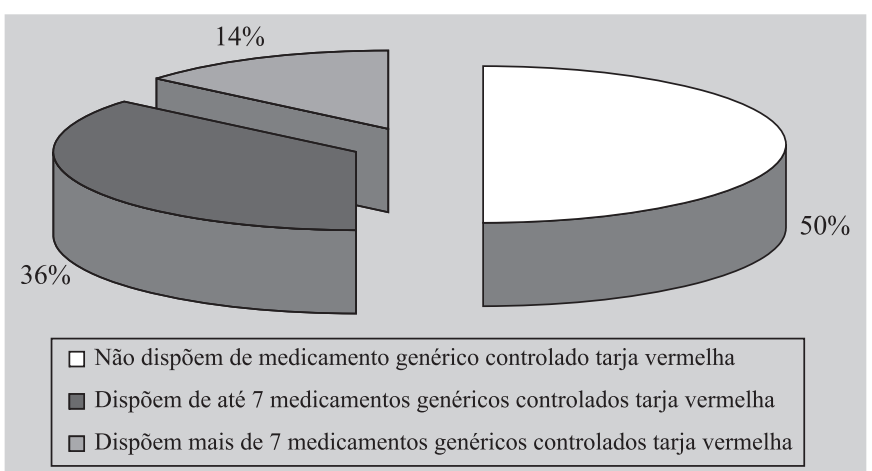

FIGURA 2 - Disponibilidade de medicamentos genéricos controlados de tarja vermelha em farmácias e drogarias no município de Maringá-PR, no período de 30/10 a 22/11 de 2002.

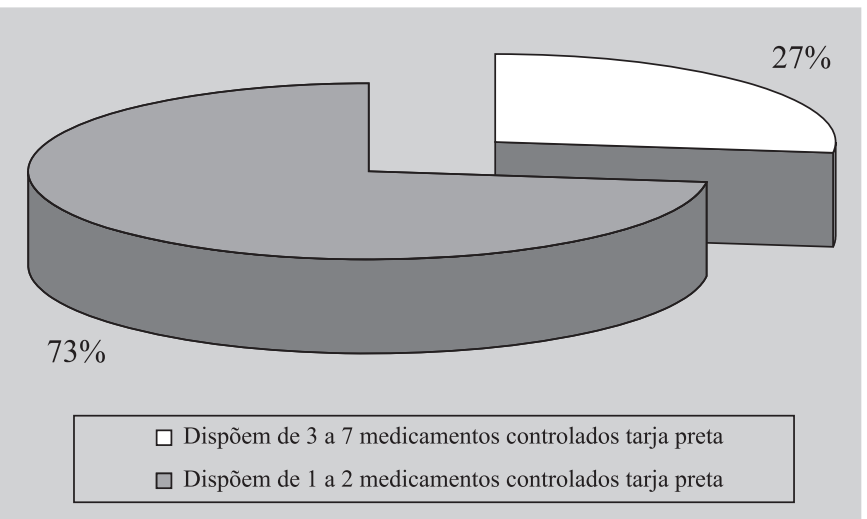

FIGURA 3 - Disponibilidade de medicamentos genéricos controlados de tarja preta em farmácias e drogarias do município de Maringá-PR, no período de 30/10 a 22/11 de 2002.

$42 \%$ mais baratos do que os de referência. Em relação aos similares, os genéricos são, em média, 15\% mais baratos. Observa-se, também, que os medicamentos similares são, em média, $24 \%$ mais baratos que os de referência (Tabela I).

A Tabela I mostra que as maiores diferenças percentuais entre os preços dos medicamentos de referência e os genéricos, para os medicamentos não sujeitos a controle especial, foram, em ordem decrescente, para o mebendazol (59\%), citrato de tamoxifeno, cloridrato de ranitidina e besilato de anlodipino (com $57 \%$ cada ) e amoxicilina (56\%). As menores diferenças percentuais para esta categoria foram para o cloridrato de amiodarona (3\%), nitrato de miconazol (22\%) e ácido acetilsalicílico e cloridrato de diltiazem (com $24 \%$ cada ).

Os genéricos, hidróxido de alumínio e o sulfato de neomicina + bacitracina, foram os medicamentos mais baratos em relação aos similares, com diferença percentual de $60 \%$ e $52 \%$, respectivamente. Alguns simi- lares, no entanto, foram mais baratos que os genéricos: o piroxicam e o diclofenaco sódico similares foram $76 \% \mathrm{e}$ $48 \%$, respectivamente, mais baratos que os seus genéricos. Ainda, conforme a Tabela I, verifica-se que o piroxicam, o diclofenaco sódico e o besilato de anlodipino foram os medicamentos similares mais baratos em relação aos de referência, enquanto os similares do hidróxido de alumínio e do sulfato de neomicina + bacitracina foram os mais caros em relação aos de referência.

Segundo Basile e Zanini (1999), medicamentos, como todos os demais produtos, também sofrem influência da propaganda, sendo habitual que médicos e pacientes conheçam apenas a marca do medicamento (nome comercial). Sem saber o nome genérico do produto o consumidor não consegue saber quais são os concorrentes. Essa influência da propaganda explica porque existe grande diferença de preço entre produtos de marcas diferentes e com o mesmo nome genérico. É esta a principal vantagem da utilização de nomes genéricos: torna-se possível a identificação de produtos similares (com a mesma substância ativa), permitindo a escolha entre diversos produtores. Aumenta-se, assim, a concorrência e o preço final ao consumidor torna-se mais barato.

$\mathrm{Na}$ análise dos medicamentos genéricos de venda controlada de tarja vermelha (Tabela II), verifica-se que eles são, em média, 36\% mais baratos do que os de referência e $12 \%$ mais baratos que os similares. Na comparação dos preços dos similares em relação aos de referência, observa-se que os primeiros são, em média, $26 \%$ mais baratos que os últimos.

Na Tabela II, observa-se, ainda, que os medicamentos genéricos mais baratos em relação aos de referência foram a carbamazepina (54\%) e o cloridrato de tramadol $(52 \%)$. Para o cloridrato de selegilina, o genérico foi $4 \%$ mais caro que o de referência. Na comparação entre o preço dos medicamentos genéricos e similares, observase que o cloridrato de tramadol genérico foi $38 \%$ mais barato que o de referência; por sua vez, o cloridrato de selegilina similar foi $10 \%$ mais caro que o de referência e o cloridrato de fluoxetina similar foi $51 \%$ mais barato em relação ao de referência e a gabapentina similar, ao contrário, foi $6 \%$ mais cara que a de referência.

No grupo de medicamentos genéricos de tarja preta, observa-se que são, em média, $37 \%$ mais baratos que os de referência e $14 \%$ mais baratos que os similares. Os similares são, em média, $26 \%$ mais baratos do que os de referência (Tabela III).

Verifica-se, na Tabela III, que os medicamentos genéricos mais baratos em relação aos de referência estão representados pelo bromazepam $(45 \%)$ e pelo clonazepam (41\%). O midazolam foi o medicamento ge- 
TABELA I - Diferenças percentuais entre os preços dos medicamentos não sujeitos a controle especial nas especialidades farmacêuticas genéricas, de referência e similares

\begin{tabular}{|c|c|c|c|}
\hline Princípio Ativo & $\begin{array}{l}\text { Diferença entre } \\
\text { referência e o } \\
\text { genérico }(\%)\end{array}$ & $\begin{array}{c}\text { Diferença entre } \\
\text { o similar e o } \\
\text { genérico }(\%)\end{array}$ & $\begin{array}{c}\text { Diferença entre } \\
\text { o referência e o } \\
\text { similar }(\%)\end{array}$ \\
\hline Aciclovir (creme, $10 \mathrm{~g}$ ) & 50 & 18 & 39 \\
\hline Ácido acetilsalicílico (200cp, 100 mg) & 24 & 20 & 5 \\
\hline Albendazol (suspensão oral, $10 \mathrm{~mL}$ ) & 40 & 7 & 36 \\
\hline Amoxicilina (15 cápsulas, 500 mg) & 56 & 49 & 14 \\
\hline Ampicilina $(12 \mathrm{cp}, 500 \mathrm{mg})$ & 33 & 39 & -10 \\
\hline Azitromicina $(3 \mathrm{cp}, 500 \mathrm{mg})$ & 41 & 27 & 19 \\
\hline Besilato de anlodipino $(20 \mathrm{cp}, 10 \mathrm{mg})$ & 57 & -2 & 58 \\
\hline Cefalexina $(10 \mathrm{cp}, 500 \mathrm{mg})$ & 54 & 43 & 20 \\
\hline Cetoconazol (creme, $30 \mathrm{~g}$ ) & 36 & 22 & 19 \\
\hline Citrato de tamoxifeno ( $30 \mathrm{cp}, 10 \mathrm{mg}$ ) & 57 & 28 & 41 \\
\hline Claritromicina (10 cp, $500 \mathrm{mg})$ & 45 & 28 & 23 \\
\hline Cloridrato de ambroxol ( xarope ad., $120 \mathrm{~mL}$ ) & 32 & 34 & -3 \\
\hline Cloridrato de amiodarona (15 cp, $200 \mathrm{mg})$ & 3 & -2 & 5 \\
\hline Cloridrato de ciprofloxacino (10 cp, $250 \mathrm{mg}$ ) & 48 & -4 & 50 \\
\hline Cloridrato de clobutinol (xarope, $120 \mathrm{~mL}$ ) & 32 & 43 & -19 \\
\hline Cloridrato de diltiazem ( 20 cápsulas, $90 \mathrm{mg}$ ) & 24 & - & - \\
\hline Cloridrato de doxiciclina $(15 \mathrm{cp}, 10 \mathrm{mg})$ & 32 & -7 & 37 \\
\hline Cloridrato de lincomicina (amp, $300 \mathrm{mg} / \mathrm{mL}$ ) & 46 & 5 & 43 \\
\hline Cloridrato de metformina $(10 \mathrm{cp}, 500 \mathrm{mg})$ & 31 & 22 & 11 \\
\hline Cloridrato de metoclopramida (gotas, $10 \mathrm{~mL}$ ) & 42 & 41 & 2 \\
\hline Cloridrato de ranitidina $(20 \mathrm{cp}, 150 \mathrm{mg})$ & 57 & 27 & 42 \\
\hline Cloridrato de verapamil ( $30 \mathrm{cp}, 80 \mathrm{mg}$ ) & 38 & 12 & 30 \\
\hline Cromoglicato dissódico (gotas, $5 \mathrm{~mL}$ ) & 37 & 16 & 25 \\
\hline Dexametasona (elixir, $120 \mathrm{~mL}$ ) & 51 & 15 & 43 \\
\hline Diclofenaco sódico (20 cp, $50 \mathrm{mg})$ & 51 & -48 & 67 \\
\hline Finasterida (30 cp, $5 \mathrm{mg})$ & 52 & - & - \\
\hline Fumarato de cetotifeno (xarope, $100 \mathrm{~mL}$ ) & 43 & - & - \\
\hline Furoato de mometasona (creme, $20 \mathrm{mg}$ ) & 46 & - & - \\
\hline Furosemida (20 cp, $40 \mathrm{mg})$ & 35 & -30 & 50 \\
\hline Hidróxido de alumínio (suspensão, 240 mL) & 42 & 60 & -60 \\
\hline Maleato de dexclorfeniramina + betametasona(xarope, $120 \mathrm{~mL}$ ) & 40 & $-0,5$ & 41 \\
\hline Maleato de enalapril (30 cp, $10 \mathrm{mg})$ & 40 & 1 & 39 \\
\hline Mebendazol (suspensão, $30 \mathrm{~mL}$ ) & 59 & 49 & 20 \\
\hline Metildopa (30 cp, $250 \mathrm{mg}$ ) & 33 & 32 & 2 \\
\hline Mupirocina (creme, $15 \mathrm{~g}$ ) & 43 & 35 & 12 \\
\hline Nifedipino (30 cp, $10 \mathrm{mg})$ & 46 & 2 & 45 \\
\hline Nimesulida (12 cp, $100 \mathrm{mg})$ & 40 & 8 & 35 \\
\hline Nitrato de miconazol (creme, $80 \mathrm{~g}$ ) & 22 & 23 & $-0,5$ \\
\hline Norfloxacino (14 cp, $400 \mathrm{mg})$ & 46 & 3 & 44 \\
\hline Paracetamol (gotas, $15 \mathrm{~mL}$ ) & 49 & 10 & 43 \\
\hline Pentoxifilina (20 cp, $400 \mathrm{mg})$ & 37 & 18 & 23 \\
\hline Piroxicam (15 cp, $20 \mathrm{mg})$ & 54 & -76 & 74 \\
\hline Prednisolona (10 cp, $20 \mathrm{mg})$ & 32 & -19 & 43 \\
\hline Sinvastatina $(30 \mathrm{cp}, 10 \mathrm{mg})$ & 53 & -7 & 56 \\
\hline Sulfametaxazol + trimetoprima $(10 \mathrm{cp})$ & 42 & 27 & 21 \\
\hline
\end{tabular}


TABELA I - continuação

\begin{tabular}{lccc}
\hline & $\begin{array}{c}\text { Diferença entre } \\
\text { o referência e o } \\
\text { genérico } \mathbf{( \% )}\end{array}$ & $\begin{array}{c}\text { Diferença entre } \\
\text { o similar e o } \\
\text { genérico (\%) }\end{array}$ & $\begin{array}{c}\text { Diferença entre } \\
\text { o referência e o } \\
\text { similar (\%) }\end{array}$ \\
\hline Sulfato de neomicina + bacitracina (pda, $50 \mathrm{~g})$ & 42 & 52 & -22 \\
Sulfato de salbutamol (xarope, $120 \mathrm{~mL})$ & 50 & 47 & 7 \\
Sulfato de terbutalina (xarope, $100 \mathrm{~mL})$ & 33 & -3 & 35 \\
Tobramicina (gotas, $5 \mathrm{~mL})$ & 42 & 26 & 22 \\
Média & $\mathbf{4 2}$ & $\mathbf{1 5}$ & $\mathbf{2 4}$ \\
\hline
\end{tabular}

$\mathrm{cp}=$ comprimido; $\mathrm{amp}=$ ampola; $\mathrm{ad}=$ adulto; $\mathrm{pda}=$ pomada

nérico com a menor diferença percentual entre o genérico e o de referência (29\%). O lorazepam e o alprazolam são os medicamentos genéricos com a maior diferença percentual em relação aos similares, sendo, respectivamente, $29 \%$ e $17 \%$ mais baratos. O diazepam genérico foi $1,97 \%$ mais caro que o seu correspondente similar. O diazepam e o bromazepam similares foram, respectivamente, $39 \%$ e $36 \%$ mais baratos que os de referência, enquanto que o lorazepam foi o medicamento similar com a menor diferença percentual em relação ao de referência, sendo $10 \%$ mais barato.

Estudos realizados em âmbito nacional revelam que os medicamentos genéricos são, em média, $40 \%$ mais baratos que os medicamentos de referência, conforme publicado pelo Conselho Regional de Farmácia do Estado de
São Paulo (CRF/SP, 2003). No presente trabalho, a análise conjunta dos três grupos de medicamentos mostrou que as especialidades genéricas foram $40 \%$ mais baratas que as de referência. Assim, o resultado obtido nesta pesquisa foi igual ao da média nacional. No entanto, a queda de preços dos medicamentos genéricos não se deu de forma homogênea para os três grupos de medicamentos estudados. Os medicamentos genéricos sujeitos a controle especial, no seu conjunto, tiveram queda de preços menor que a dos medicamentos genéricos sem controle especial, respectivamente, 36 e $42 \%$.

Em fevereiro de 2001, após um ano de implantação dos genéricos, um levantamento da ANVISA, em farmácias de 11 capitais, mostrou que algumas especialidades genéricas chegavam a ser até $62 \%$ mais baratas que os

TABELA II - Diferenças percentuais entre os preços dos medicamentos controlados de tarja vermelha nas especialidades farmacêuticas genéricas, de referência e similares

\begin{tabular}{|c|c|c|c|}
\hline Princípio Ativo & $\begin{array}{c}\text { Diferença entre } \\
\text { o referência e o } \\
\text { genérico }(\%)\end{array}$ & $\begin{array}{c}\text { Diferença entre } \\
\text { o similar e o } \\
\text { genérico }(\%)\end{array}$ & $\begin{array}{c}\text { Diferença entre } \\
\text { o referência e o } \\
\text { similar }(\%)\end{array}$ \\
\hline Ácido valpróico (30 cápsulas, 250 mg) & 19 & - & - \\
\hline Carbamazepina (10 cp, $200 \mathrm{mg})$ & 54 & 29 & 35 \\
\hline Carbidopa/levodopa (30 cp, $25 \mathrm{mg}+250 \mathrm{mg}$ ) & 35 & - & - \\
\hline Cloridrato de biperideno ( $80 \mathrm{cp}, 2 \mathrm{mg})$ & 41 & 5 & 38 \\
\hline Cloridrato de buspirona (20 cp, $10 \mathrm{mg}$ ) & 35 & - & - \\
\hline Cloridrato de fluoxetina (14 cp, $20 \mathrm{mg}$ ) & 49 & -5 & 51 \\
\hline Cloridrato de mianserina $(20 \mathrm{cp}, 30 \mathrm{mg})$ & 34 & - & - \\
\hline Cloridrato de paroxetina ( $15 \mathrm{cp}, 20 \mathrm{mg}$ ) & 36 & -8 & 41 \\
\hline Cloridrato de selegilina ( $15 \mathrm{cp}, 5 \mathrm{mg})$ & -4 & -10 & 5 \\
\hline Cloridrato de sertralina $(30 \mathrm{cp}, 50 \mathrm{mg})$ & 47 & - & - \\
\hline Cloridrato de tramadol (6 amp, $50 \mathrm{mg} / \mathrm{mL})$ & 52 & 38 & 22 \\
\hline Gabapentina (15 cápsulas, $300 \mathrm{mg}$ ) & 30 & 34 & -6 \\
\hline Haloperidol (gotas, $20 \mathrm{~mL}$ ) & 29 & 9 & 22 \\
\hline Moclobemida (30 cp, $150 \mathrm{mg}$ ) & 40 & - & - \\
\hline Média & 36 & 12 & 26 \\
\hline
\end{tabular}

$\mathrm{cp}=$ comprimido; $\mathrm{amp}=$ ampola 
TABELA III - Diferenças percentuais entre os preços dos medicamentos controlados de tarja preta nas especialidades farmacêuticas genéricas, de referência e similares

\begin{tabular}{lccc}
\hline Princípio Ativo & $\begin{array}{c}\text { Diferença entre } \\
\text { o referência e o } \\
\text { genérico (\%) }\end{array}$ & $\begin{array}{c}\text { Diferença entre } \\
\text { o similar e o } \\
\text { genérico (\%) }\end{array}$ & $\begin{array}{c}\text { Diferença entre } \\
\text { o referência e o } \\
\text { similar (\%) }\end{array}$ \\
\hline Alprazolam (20 cp, $0,5 \mathrm{mg})$ & 35 & 17 & 22 \\
Bromazepam $(20 \mathrm{cp}, 3 \mathrm{mg})$ & 45 & 13 & 36 \\
Clonazepam (30 cp, $2 \mathrm{mg})$ & 41 & - & - \\
Diazepam (20 cp, 10 mg) & 38 & -2 & 39 \\
Lorazepam (20 cp, 2 mg) & 36 & 29 & 10 \\
Midazolam (5 amp, 15 mg/3mL) & 29 & - & - \\
Zopiclona (20 cp, 7,5 mg) & 32 & 11 & 23 \\
Média & $\mathbf{3 7}$ & $\mathbf{1 4}$ & $\mathbf{2 6}$ \\
\hline
\end{tabular}

$\mathrm{cp}=$ comprimido; $\mathrm{amp}=$ ampola

medicamentos de marca (Jatene et al., 2002). Os mesmos autores citam que os medicamentos genéricos podem ser, em alguns casos, até $80 \%$ mais baratos que os de marca. Neste trabalho, demonstrou-se que em várias situações a diferença percentual entre os preços dos genéricos e dos medicamentos de marca foi maior que $50 \%$ (por exemplo: aciclovir, amoxicilina, besilato de anlodipino, cefalexina, citrato de tamoxifeno, cloridrato de ranitidina, dexametasona), e para o hidróxido de alumínio a diferença percentual chegou a $60 \%$.

Com base nessas informações de preços e qualidade, o consumidor pode economizar na compra de seus medicamentos, pois a diferença de preços entre medicamentos idênticos, com marcas diferentes, chega a ser mais do que o dobro (Basile, Zanini, 1999).

Após a introdução dos medicamentos genéricos no mercado brasileiro, entre os tratamentos que ficaram mais baratos estão o de diabetes, que caiu de 34 a 37\% (dependendo do medicamento adotado), o de hipercolesterolemia, cuja queda de preço variou de 39 a $57 \%$, e o de hipertensão, cerca de 53\% (CRF/SP, 2003). Neste trabalho o cloridrato de metformina na especialidade farmacêutica genérica foi $31 \%$ mais barato em relação ao medicamento de referência, valor inferior à queda de preço dos medicamentos antidiabéticos em nível nacional. A sinvastatina na forma farmacêutica genérica foi 53\% mais barata em relação ao medicamento de referência, valor condizente com os dados nacionais para os hipocolesterolemiantes. Os medicamentos genéricos para o tratamento da hipertensão encontrados nesta pesquisa (besilato de anlodipino, cloridrato de diltiazem, cloridrato de verapamil, furosemida, maleato de enalapril, metildopa e nifedipino) foram, em conjunto, 39\% mais baratos que os de referência, valor inferior aos dados nacionais.
O sucesso da política pública dos medicamentos genéricos beneficiou, especialmente, as pessoas com doenças crônicas, que consomem os medicamentos de forma contínua. Conforme Jatene et al. (2002), com a expansão do mercado dos genéricos, houve redução expressiva no custo do tratamento das seis doenças crônicas mais freqüente no País: hipertensão, diabetes, hipercolesterolemia, gota, hiperplasia da próstata e glaucoma.

Observou-se, no presente trabalho, que dos 71 medicamentos genéricos encontrados, 15 (21\%) foram mais caros que os similares (besilato de anlodipino, cloridrato de amiodarona, cloridrato de ciprofloxacino, cloridrato de doxiciclina, diclofenaco sódico, furosemida, maleato de dexclorfeniramina + betametasona, piroxicam, prednisolona, sinvastatina, sulfato de terbutalina, cloridrato de fluoxetina, cloridrato de paroxetina, cloridrato de selegilina e diazepam) e um (1\%) foi mais caro que o medicamento de referência (cloridrato de selegilina), conforme as Tabelas I, II e III. Dessa forma, apesar de, na média, os medicamentos genéricos apresentarem preço inferior aos de marca, em 16 situações isso não ocorreu.

Para os três grupos de medicamentos pesquisados, houve significativa redução dos preços dos genéricos em relação aos medicamentos de referência e aos similares, principalmente para os medicamentos não sujeitos a controle especial. Nos Estados Unidos, os medicamentos genéricos chegam ao mercado com o preço $25 \%$ inferior aos medicamentos de marca e essa queda pode atingir $60 \%$ em dois anos de comercialização (Jatene et al., 2002). $\mathrm{Na}$ Espanha, o consumo de genéricos tem crescido, mas não tanto como nos Estados Unidos, sendo que neste país o preço desses medicamentos é de 24 a $89 \%$ mais barato que os de marca (Iñesta García, 2000). Num estudo semelhante ao presente, realizado no Canadá, não se observou 
alteração significativa entre os preços dos medicamentos genéricos e os de marca (Lexchin, 2004).

Em nível mundial, a história dos medicamentos genéricos começa na década de 1960, sendo que o primeiro país a implantar política específica foi os Estados Unidos. Atualmente, mais de $70 \%$ do receituário médico americano são de genéricos. No Reino Unido, os genéricos representam $15 \%$ do mercado e na Alemanha, 30\% (Jatene et al., 2002). Provavelmente, com o decorrer do tempo, no Brasil, a aceitação e o consumo de medicamentos genéricos ocorram da mesma forma que nesses países.

A experiência internacional mostra que os maiores êxitos obtidos na promoção dos genéricos têm ocorrido nos países em que as ações são direcionadas para influenciar o comportamento de médicos e profissionais de saúde por meio de informações que comprovam a qualidade e a confiabilidade desses medicamentos (Jatene et al., 2002). Como muitos medicamentos genéricos, apesar de mais baratos não foram encontrados em farmácias e drogarias pesquisadas no presente trabalho, ações educativas junto aos profissionais de saúde, especialmente médicos e farmacêuticos, devem ser difundidas.

Uma competitividade saudável entre empresas que produzem e vendem medicamentos tem tido, nos países desenvolvidos, participação expressiva na redução de custos, desde que possa ser regulada, do ponto de vista do comprador, particularmente no que concerne à garantia de qualidade. No âmbito do serviço público no Brasil, existe farta legislação que tenta oferecer meios para regulamentação da oferta e dos processos de aquisição (Luiza, Castro, Nunes, 1999). Dessa forma, os medicamentos genéricos se inserem como ferramentas estratégicas para a diminuição dos custos e manutenção da qualidade nos serviços públicos de saúde.

Apesar do incontestável progresso na racionalização terapêutica após a liberação dos medicamentos genéricos no Brasil, é necessário que se conheça em profundidade os obstáculos reais ao pleno estabelecimento da política dos genéricos no Brasil. Conforme Carvalho et al. (2003), devem ser elaboradas estratégias eficientes que visem ultrapassar esses empecilhos para que a citada política alcance pleno êxito no âmbito social. Os autores demonstraram a preocupação do consumidor com o preço, fator determinante do acesso ao medicamento para alguns segmentos da população, seguida da qualidade e das características terapêuticas do produto. Verifica-se, assim, a preocupação dos usuários dos medicamentos com os aspectos econômicos da terapia, que podem ser proibitivos ao acesso pela população.

Um dos desafios da política de genéricos está em transmitir ao consumidor as informações necessárias para que este se torne de fato um usuário de medicamentos genéricos. O paciente tem poucas condições de escolher sozinho qual medicamento vai comprar, tornando-se dependente de opinião profissional. Dessa maneira, o principal canal de estímulo aos genéricos está na contínua educação de médicos e farmacêuticos sobre a disponibilidade dos genéricos, os princípios ativos, o diferencial de preços e a eficácia idêntica desse grupo de medicamentos.

A lei número 9787/99, publicada no DOU, regulamenta o uso do nome genérico dos medicamentos em nosso país e ameaça de tal modo os interesses econômicos das indústrias, que levantou questões quanto à similaridade de medicamentos, equivalência terapêutica e farmacêutica, padrão de prescrição, direito à substituição e outras (Cunha et al., 2002). Enquanto todos esses problemas não são resolvidos, a ANVISA não poderá fazer cumpri-la plenamente.

\section{CONCLUSÃO}

A política nacional de medicamentos, no que diz respeito aos medicamentos genéricos, representa avanço no acesso da população ao tratamento medicamentoso, uma vez que facilita a aquisição dos produtos farmacêuticos pelos usuários. Isso representaria, a longo prazo, melhora na qualidade assistencial e, conseqüentemente, na qualidade de vida dos usuários, que obteriam um medicamento de qualidade a menor custo. A diferença de preços entre os medicamentos genéricos e os de marca beneficia tanto o setor público quanto o privado, permitindo a estes a escolha de medicamento com qualidade a um menor preço. Apesar disso, na presente pesquisa, evidenciou-se a não disponibilização da maioria dos princípios ativos liberados pela ANVISA como especialidades farmacêuticas genéricas nos estabelecimentos farmacêuticos pesquisados.

\section{ABSTRACT}

\section{An evaluation of generic medicines availability in pharmacies and drugstores in Maringá (PR) and comparison of their prices in relation with the similars and the references}

The discussion about the implementation of generics has being carried trough since 1976 in Brazil. However, just in 1991 a project which proposes the implantation of generic medicines has been elaborated. The Law 9787 (called Law of the Generic medicines) has been only published in Diário Oficial da União in February $11^{\text {th }}$, 1999. The objectives of this work had been to verify generic medicine availability in pharmacies and drugstores, as well as comparing their prices in relation 
with the similars and the references. The generic availability was verified in 22 pharmacies and drugstores in Maringá city, state of Paraná, from October, $30^{\text {th }}$ to November, $20^{\text {th }}, 2002$. In a total of 222 active principles available in Brazil as generic, 71 (32\%) had been found. Those generic medicines sold not under special control are in average $42 \%$ cheaper than the reference and $15 \%$ cheaper than the similar ones. Those generic medicines with red label, sold under special control, are in average $36 \%$ cheaper than the reference and $12 \%$ cheaper than the similar ones. The generic medicines with black label are $37 \%$ cheaper than the reference and $14 \%$ cheaper than the similars ones.

UNITERMS: Generic medicines. Medicines/prices. Similar medicines. Reference medicines. Public health.

\section{AGRADECIMENTO}

Agradecemos a Laura Akemi Côrtes Massunari, pelas sugestões na redação do texto final e na elaboração do abstract.

\section{REFERÊNCIAS BIBLIOGRÁFICAS}

ANVISA. Lista dos centros de bioequivalência certificados pela Anvisa para a realização de testes de biodisponibilidade e bioequivalência. Disponível em: [http:/ /www.anvisa.gov.br/inspecao/bioequivalencia/index.htm] Acesso em : 10 de janeiro de 2005.

BARROS, J.A.C. Propaganda de Medicamentos: atentado à saúde? Sobravime-Hucitec, p. 222, São Paulo, 1995.

BARROS, J.A.C. A Atuação dos balconistas em farmácias. J. Bras. Med., v.73, n.2, p.120-124, São Paulo, 1997.

BASILE, A.C.; ZANINI, A.C. Dicionário de Medicamentos Genéricos Zanini-Oga. São Roque: IPEX Editora, 1999. $311 \mathrm{p}$.

BRANDÃO, A. Governador de Goiás sai em defesa dos genéricos . Pharm. Bras.,v. 7, n. 23, p.46-47, São Paulo, 2000.

BRASIL, 1999. Lei n. ${ }^{\circ}$ 9787, de 10 de fevereiro de 1999. Altera a lei n. ${ }^{\circ} 6360$, de 23 de setembro de 1976, que dispõe sobre a vigilância sanitária, estabelece o medicamento genérico, dispõe sobre a utilização de nomes genéricos em produtos farmacêuticos e dá outras providências. Diário Oficial da República Federativa do Brasil, DF, 11 fev. 1999. Seção 1, 4 p.
BRASIL, 2000. Resolução no 41, de 28 de abril de 2000. Estabelece critérios para a aceitação das unidades que realizam ensaios de equivalência farmacêutica, biodisponibilidade e bioequivalência em medicamentos. Diário Oficial da República Federativa do Brasil, Brasília, DF, 3 mai. 2000. Seção 1. 14 p.

BRASIL, 2001. Resolução nº 47, de 28 de março de 2001. Dispõe sobre as embalagens de Medicamentos Genéricos. O uso de faixa amarela para genéricos. Diário Oficial da República Federativa do Brasil, Brasília, DF, 5 abr. 2001. Seção 1. p. 20-32.

BRASIL, 2003. Resolução n 134, de 29 de maio de 2003. Dispõe sobre a adequação dos medicamentos já registrados. Diário Oficial da República Federativa do Brasil, Brasília, DF, 2 jun. 2003. Seção 1. 14 p.

CARVALHO, M.C.R.D.; CAMPOS, M.N.; CRUS, M. M.C. et al. Vai um genérico aí? In: Congresso Brasileiro de Medicamentos Genéricos, 4,Goiânia, 2003. Revista do $4^{\circ}$ Congresso Brasileiro de Medicamentos Genéricos. São Paulo, 2003.p. 9.

CHIRAC, P. Generics: neither Angel nor demons. Rev. Presc., v.12, n.118, p.249-253, 1992.

CONSELHO REGIONAL DE FARMACIA DO ESTADO DE SÃO PAULO. Fabricantes de genéricos vão à luta. Rev. do Farm., n. 64, p. 22-25, São Paulo, jun.jul. 2003.

CUNHA, M.C.N.; ZORZATTO, J.R.; CASTRO, L.L.C. Avaliação do uso de medicamentos na Rede Pública Municipal de Saúde de Campo Grande/MS. Rev. Bras. Ciênc. Farm., v. 38, n. 2, abr.jun. 2002.

INDIFARMA. Índice de preços de medicamentos (Inditec). Maringá: INDITEC Índices Técnicos e Processamento Ltda, 2003.90 p.

IÑESTA GARCÍA, A. Genéricos y precios de referencia, rango de precios y fuentes de información. Ars Pharma., v. 41, n.3, p. 365-378, 2000.

JATENE, A.D.; PINA, F.A.L.; CARAVANTE JUNIOR, F.P.G. et al. Manual médico: medicamentos genéricos. São Paulo: Lemos Editorial, 2002. 141 p.

LEXCHIN, J. The effect of generic competition on the price of brand-name drugs. Health Policy, v. 68, n. 1, p. 47-54, apr. 2004. 
LUIZA, V.L.; CASTRO, C.G.S.O.; NUNES, J.M. Aquisição de medicamentos no setor público: o binômio qualidade- custo. Cad. Saúde Pública, v. 15, n. 4, p. 769$776,1999$.

LYRA JUNIOR, D.P., OLIVEIRA, M. A C., AMORIM, E. L. C. Aconselhamento ao paciente: necessidade curricular. Infarma, v.7, n.1/2, p.20-21, 1999.

MARQUES, D.C. Uso racional de medicamentos: alguns pontos para reflexão. $D M G, \mathrm{n} .20, \mathrm{p} .2,2001$.

MELLA, E.A.C., VENDRAMETTO, M. C., MELLA Jr, S. E., VICENTE, J. G. Avaliação sobre o conhecimento e utilização dos medicamentos genéricos por acadêmicos de uma instituição de ensino superior. Infarma, v.14, n.11/ 12,p.49-52, 2002.

SAX, B.W. What you should know about generic drugs. Pharm. Times, n.2, p.19-24, 2000.

SANTANA, A.D., LYRA JUNIOR, D. P., NEVES, S. J. F. Qualidade da informação farmacêutica na dispensação dos medicamentos genéricos. Pharm. Bras., n.39, p.84-86, 2003.
SANTOS, J.S. O que nos ensinam os genéricos. Pharm. Bras., n.19, p.3, 2000.

SERRA, J. Serra pede a médicos que prescrevam genéricos. Pharm. Bras., n.19, p.8, 2000a.

SERRA, J. Farmácia pode fechar se não vender genéricos. Pharm. Bras., n.19, p.7, 2000 b.

VALENTE, V.; STORPIRTIS, S. A estratégia para consolidação da política nacional de medicamentos. Fárm. Medicam., v. 2, n.11, p.32-33, 2001.

ZANINI, A.C. Genéricos - o médico decide. Dicionário de medicamentos genéricos. Instituto de defesa do usuário de medicamentos e CRF, DF. São Paulo: IPEX, 1999, p.4-5.

Recebido para publicação em 10 de março de 2004. Aceito para publicação em 22 de julho de 2005. 